\title{
REVIEW
}

\section{Clinical pathology accreditation: standards for the medical laboratory}

\author{
D Burnett, C Blair, M R Haeney, S L Jeffcoate, K W M Scott, D L Williams
}

J Clin Pathol 2002;55:729-733

This article describes a new set of revised standards for the medical laboratory, which have been produced by Clinical Pathology Accreditation (UK) Ltd (CPA). The original standards have been in use since 1992 and it was recognised that extensive revision was required. A standards revision group was established by CPA and this group used several international standards as source references, so that the resulting new standards are compatible with the most recent international reference sources. The aim is to make the assessment of medical laboratories as objective as possible in the future. CPA plans to introduce these standards in the UK in 2003 following extensive consultation with professional bodies, piloting in selected laboratories, and training of assessors.

See end of article for authors' affiliations

Dr D Burnett, Lindens Lodge, Bradford Place, Pernarth CF64 1LA, UK; davidburnett3@aol.com Accepted for publication 12 May 2002 ince its incorporation in January 1992, Clini-

S cal Pathology Accreditation (UK) Ltd (CPA) has, with some modification, used standards ${ }^{1}$ based on the laboratory sections of the Canadian, Australian, and UK standards for healthcare. In 1996, the first drafts of a new international standard, ISO 15189 Quality management in the medical laboratory were circulated. As a result of this development, the board of CPA commissioned an internal report that made two main recommendations. First, that in the light of changes taking place in international standards, a standards revision group (SRG) should be established to review existing standards and, second, that CPA should seek to operate in conformity with international standards such as ISO guide $58^{2}$ or Standards for health care accreditation bodies. ${ }^{3}$

The SRG first met in September 1998 and draft standards went to the shareholders of CPA for consultation in April 2000. After this consultation and revision the new standards were approved by the board of CPA on 8 December 2000 and published on the CPA website in January $2001 .^{4}$

It is accepted that the introduction of these new standards will affect the assessment (inspection) process. Assessors will need to understand and be able to assess a quality management system, in addition to the professional aspects of normative document. Such documents are subdivided into normative document. Such documents are subdivided into
clauses that are equivalent to standards as presented by CPA
* Internationally the word "standard" is used to denote the functioning of a medical laboratory. This will require retraining of the current inspectors and the recruitment of new ones.

\section{APPROACH TO THE REVISION}

Members of the SRG were drawn from the main laboratory professions and from all the major disciplines of pathology. This main group met on nine occasions with a subgroup undertaking editorial work. At the outset the SRG established several guiding principles. The new standards were drafted to be in conformity with several documents that were either published ${ }^{5}$ or in the process of being revised and published..$^{7-9}$ These documents were adopted as source references (section 3).

\section{"It is accepted that the introduction of these new standards will affect the assessment (inspection) process"}

It was also agreed that a convention used in writing international standards would be adopted; namely, that each standard/clause* should have a title, that the use of the word "shall" denotes a requirement and "should" a recommendation. Terms used in a technical sense would be defined and a bibliography of informative references provided.

Experience with the original CPA standards has shown that it is not always easy to find the evidence against which to judge compliance and that this leads to inconsistencies in the assessment process. It was agreed that the new standards should be written, as far as possible, with a clarity that would enable compliance with each standard to be unequivocally verified during an onsite assessment (section 6).

\section{SOURCE REFERENCES}

The ISO standards used as source documents by the SRG in drafting new standards come from two distinct lineages. The first has its origin in quality management system (QMS) standards published in 1987 by the British Standards Institute. In 1994 these were further developed as the much quoted and extensively used ISO 9000 series of QMS standards, which have now been revised and published as ISO 9001:2000 Quality management systems-requirements, ${ }^{9}$ with

Abbreviations: CPA, Clinical Pathology Accreditation Ltd; ISO, International Organisation for Standardisation; QMS, quality management system; SRG, standards revision group 


\section{A4 Quality management system}

A quality management system provides the integration of organisational structure, procedures, processes and resources needed to fulfil a quality policy and thus meet the needs and requirements of users.

A 4.1 Laboratory management shall establish a quality management system.

A 4.2 Roles, responsibilities, and authority of all personnel shall be defined to ensure the establishment, implementation, and maintenance of the quality management system.

A 4.3 Laboratory management shall be responsible for: (a) setting quality objectives and undertaking quality planning (A5) (b) preparing a quality manual (A6) (c) appointing a quality manager (however named) (A7) (d) establishing a procedure for document control (A8) (e) establishing a procedure for control of process and quality records (A9) (f) establishing a procedure for control of clinical material (A10) (•) conducting a management review (All).

Cross references

- ISO/FDIS 15189

- ISO/IEC 170025:2000

- ISO/DIS 9001 (E)

- EC4 Essential Criteria
4.2
4.2
5.5
Quality management system
Quality system
Administration (of a quality system)
Quality strategy of the laboratory

Figure 1 Clinical Pathology Accreditation standard: A4 Quality management system. associated documents. ${ }^{11}{ }^{12}$ This latest revision presents its requirements in the context of a process based quality management system and has had a considerable impact on the way the new CPA standards are presented.

QMS standards are generic in nature and therefore applicable to many situations. They are used for certification, which is defined in ISO/IEC guide $2^{13}$ as the "procedure by which a third party gives written assurance that a product, process or service conforms to specific requirements". Certifying bodies are concerned with auditing the quality management systems of companies or facilities. If the company or organisation is manufacturing a product or providing a service, the assessment looks at the documentation and functioning of the quality management system, not at the competence (technical resources) required to manufacture the product or provide the service.

The second lineage focuses on requirements of calibration and testing laboratories. These standards have their origins in the various editions of ISO/IEC guide 25 . The third revision ${ }^{14}$ of this guide was started in 1988 and completed in 1990. Work on the European standard, EN 45001:1989 General criteria for the operation of testing laboratories, ${ }^{15}$ which is based on ISO/ IEC guide 25, was started in 1987 and completed in 1989. The replacement for EN45001:1989 was published in December 1999 as ISO/IEC 17025:1999 Requirements for testing and calibration laboratories. ${ }^{8}$ Although technical requirements form a major part of these documents, the influence of ISO 9000 QMS standards can be clearly seen.

ISO/IEC 17025:1999 is a generic standard used for the accreditation of many different types of testing and calibration laboratories. The words certification and accreditation in common English usage are interchangeable; however, in their technical usage, accreditation is distinguished from certification and defined as a "procedure by which an authoritative body gives formal recognition that a body or person is competent to carry out specific tasks". The requirements of ISO/IEC 17025:1999 are contained in two main paragraphs: “4 Quality management" and "5 Technical resources". When a laboratory seeks accreditation, the assessment is not limited to the documentation and functioning of the quality management system, but also examines whether the laboratory has the competence and technical resources to carry out specific tasks.

In addition to these generic standards, work started in 1996 on an international standard, ISO 15189, intended for specific use by medical laboratories, and is now in the final stages of consultation before publication. In the early stages of its preparation, two papers ${ }^{56}$ prepared for publication by the European Community Confederation of Clinical Chemists (EC4), referred to collectively in this paper as the EC4 Essential criteria for quality systems in laboratory medicine, were used in the development of ISO 15189.

"The new CPA standards draw upon the best and most up to date material from their reference sources"

In a recent publication, ${ }^{16}$ the essential criteria have been proposed as guidance notes to both ISO/IEC 17025:1999 and ISO/FDIS 15189 and contain a detailed cross reference table to both international standards. The CPA standards are at present cross referenced to the August 2000 FDIS version of the ISO 15189 standard, entitled "Quality management in the medical laboratory". The current version has been retitled "Medical laboratories-particular requirements for quality and competence" ${ }^{\prime \prime}{ }^{17}$ a title that better reflects its intended use as a standard for use by accreditation bodies. It is a standard concerned with both the requirements for quality management and the competence of the laboratory to undertake a specified task. The new CPA standards draw upon the best and most up to date material from these reference sources.

\section{PRESENTATION OF THE REVISED STANDARDS}

The new standards are presented in a document entitled "standards for the medical laboratory" ${ }^{4}$ in a similar format to an international standard. Sections on scope and purpose, references, terms, and definitions are followed by eight sections of standards that retain the alphanumeric labelling of individual standards familiar to existing CPA accredited laboratories.

In the new format each individual standard has a defined structure, namely:

- a unique alphanumeric identifier

- a title

- a descriptive passage that is intended to put the standard in context (in italics)

- the clauses of the standard, each with a unique alphanumeric label 


\begin{tabular}{|ll|}
\hline Sections of CPA revised standards & Corresponding sections of ISO 9001:2000 \\
\cline { 2 - 2 } A Organisation and quality management system & $\begin{array}{l}4 \text { Quality management system } \\
5 \text { Management responsibility }\end{array}$ \\
\cline { 2 - 2 } $\begin{array}{l}\text { B Personnel } \\
\text { C Premises and environment }\end{array}$ & \multicolumn{2}{c}{6 Resource management } \\
D Equipment, information systems, and reagents \\
\cline { 2 - 2 } $\begin{array}{ll}\text { E Pre examination processes } \\
\text { F Examination processes }\end{array}$ \\
F Post examination processes & 7 Product realisation \\
H Evaluation and quality assurance & \\
\hline
\end{tabular}

Figure 2 Sections of the revised Clinical Pathology Accreditation standards relative to ISO 9001:2000
- explanatory notes where appropriate

- cross references to source material.

Figure 1 shows an example of this structure.

\section{THE RELATION BETWEEN SECTIONS OF STANDARDS: A PROCESS BASED MODEL}

The CPA's original 44 standards were arranged into six sections but the relation between the sections was not explicit. The eight sections of the new standards are shown in column 1 of fig 2. The second column shows the corresponding sections of ISO 9001:2000.

Figure 3 illustrates the dynamic relation between the sections of standards and is based on the process based model of quality management developed in ISO 9001:2000. In this relation, the organisation of the laboratory and its quality management system (section A) uses resources (sections B, C, and D) to undertake pre examination, examination, and post examination processes (sections $\mathrm{E}, \mathrm{F}$, and $\mathrm{G}$ ), which are continually monitored and evaluated (section $\mathrm{H}$ ). These revised CPA standards are perhaps the first to be used by an accreditation body in which the individual standards, or clauses, are arranged in a manner that fully integrates the quality management and technical competence requirements for a medical laboratory.

\section{THE CONTENT OF THE NEW STANDARDS}

Certain sections of the revised standards introduce concepts that will be unfamiliar to some laboratories. In section A, the first standard $(\mathrm{Al})$ defines the organisation and management requirements of a department, whereas the second standard (A2) specifically deals with the needs and requirements of users. It then goes on to consider aspects of a quality management system and particular emphasis is placed upon the importance of:

- A quality manual as an index to documentation.

- The appointment of a quality manager who oversees, as part or the whole of their job, the implementation and maintenance of the quality system.

- A systematic approach to document control.

- An annual management review to ensure the continuation of the service at a level that meets the needs and requirements of users.

It is suggested that a copy of the annual management review should be sent to CPA as a means of monitoring continuing compliance with the standards between assessments.

Sections B, C, and D deal with the resources of a laboratory and include material that is largely familiar to CPA applicants and encompass the management of personnel, premises, environment, equipment, information systems, and reagents.

Sections E, F, and G deal with pre examination, examination, and post examination processes and present familiar material in a logical and structured format. The term examination is used rather than test or analysis because it is used internationally and best describes the activity of all disciplines in pathology.

"Certain sections of the revised standards introduce concepts that will be unfamiliar to some laboratories"

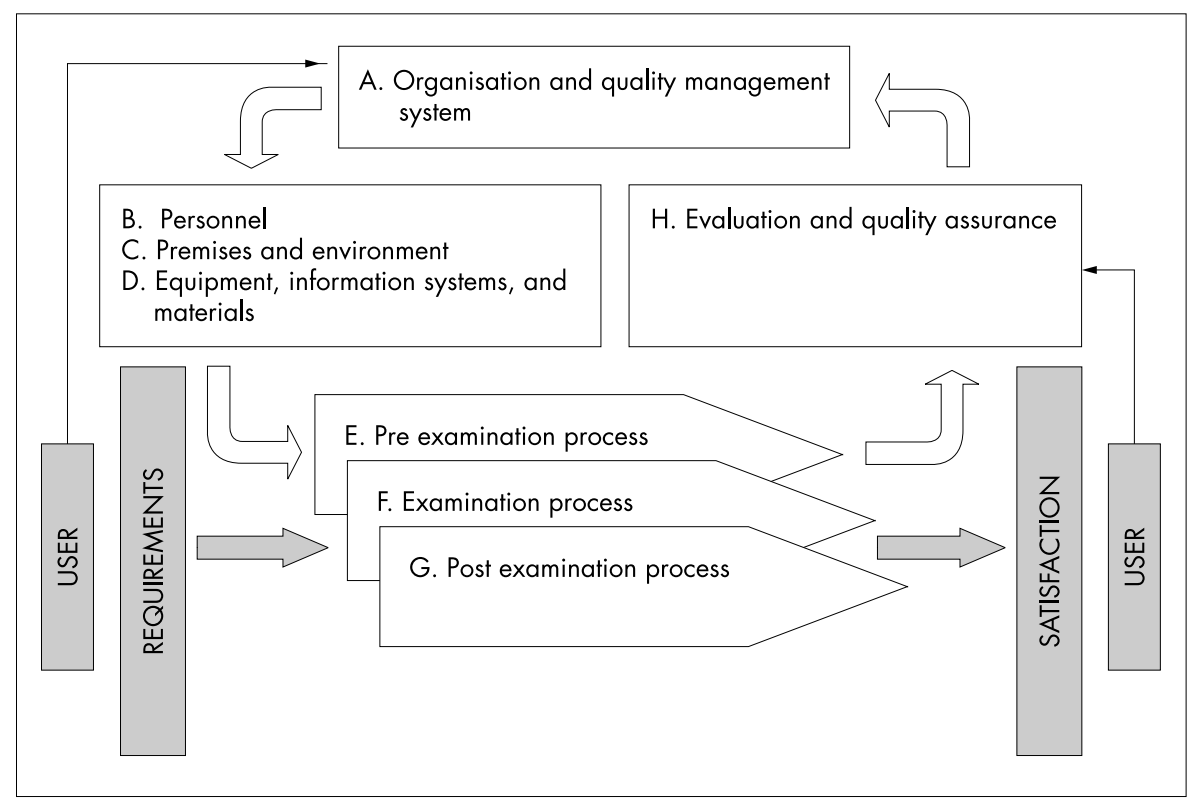

Figure 3 Process based relation between sections of the revised Clinical Pathology Accreditation standards. 


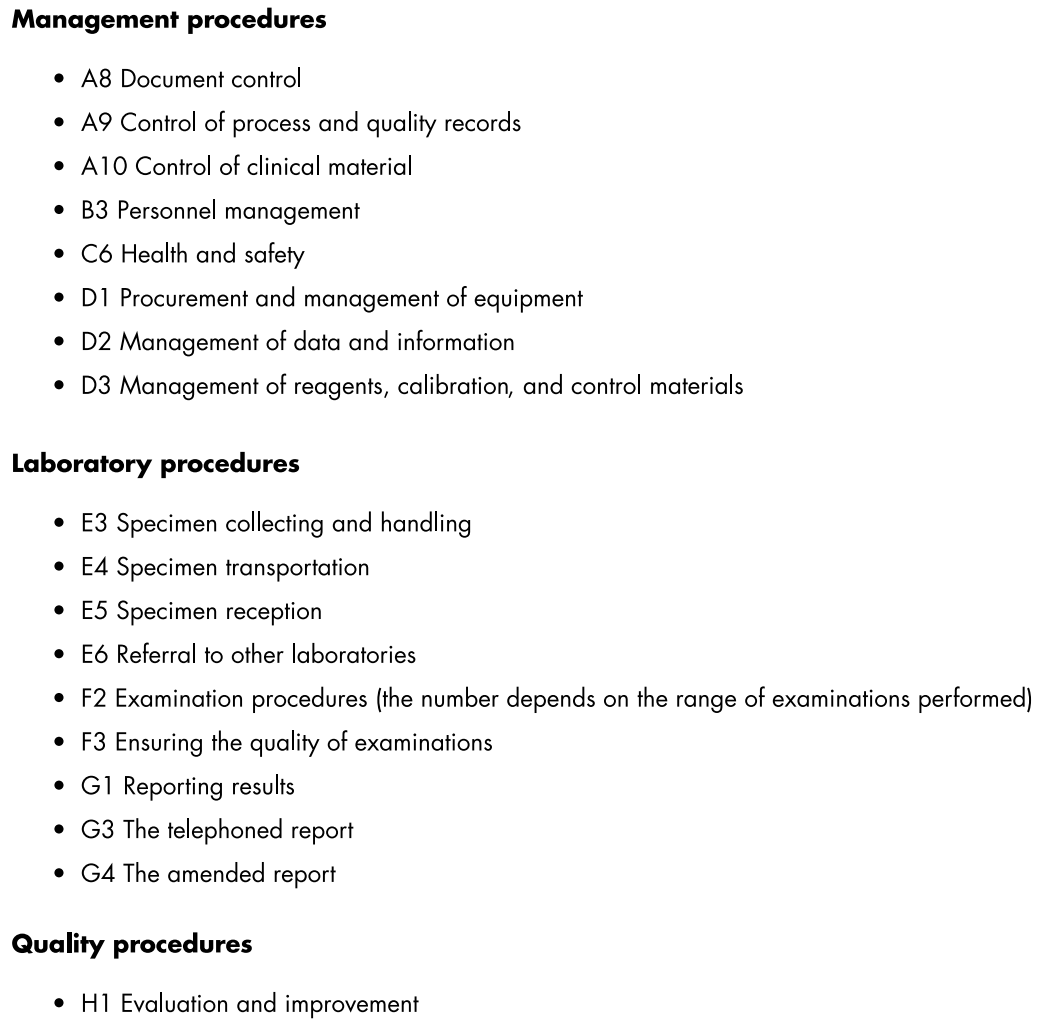

- D1 Procurement and management of equipment

- D2 Management of data and information

- D3 Management of reagents, calibration, and control materials

\section{Laboratory procedures}

- E3 Specimen collecting and handling

- E4 Specimen transportation

- E5 Specimen reception

- E6 Referral to other laboratories

- F2 Examination procedures (the number depends on the range of examinations performed)

- F3 Ensuring the quality of examinations

- G1 Reporting results

- G3 The telephoned report

- G4 The amended report

\section{Quality procedures}

- H1 Evaluation and improvement

Figure 4 Clinical Pathology Accreditation standards requiring procedures.
Section $\mathrm{H}$ deals explicitly with quality assurance and evaluation. This includes the monitoring of the performance of the quality management system in terms of user satisfaction, complaints, and internal audit. It also covers the performance of examinations, including both internal audit and external quality assessment. Central to the new standards is an emphasis on the importance of continual improvement processes.

\section{OBJECTIVE ASSESSMENT}

In section 2, mention is made that "the SRG has sought to write these new standards in such a way that compliance with each standard should be unequivocally verifiable during an onsite visit".

In the case of the standards shown in fig 4, the first step towards evidence of compliance with the particular standard is the inspection of the required written procedure. Many procedures require the use of forms (hard copy or electronic) upon which records are made. These records are the evidence that the processes described in the procedure are being performed correctly. For example, a procedure for personnel management will require several different forms upon which records can be made. Examples would include forms to record staff induction and joint annual review.

In addition to the forms and records arising from the implementation of procedures, other evidence is availablefor example, in minutes of meetings, reports from participation in external quality assessment schemes, health and safety, and fire inspections.

\section{INTRODUCTION OF THE NEW STANDARDS}

The introduction of revised standards such as these requires a clear timetable and an educational programme. During 2001, a series of one day educational meetings were planned and organised by an educational working group. These meetings were held in different locations throughout the UK and were attended by current inspectors and staff from CPA accredited laboratories. At these meetings presentations were given by a small group of speakers who had been involved in writing the new standards, and attendees at these meetings received copies of the draft templates for a quality manual, a procedure, and a vertical audit report, in addition to guidance documents on an approach to documentation in the medical laboratory, a quality policy for the medical laboratory, a job description for a quality manager in the medical laboratory, and an approach to audit in the medical laboratory.

This documentation and the accompanying templates are intended to be indicative of a possible approach to the management and structuring of medical laboratory documentation. They are not intended to be prescriptive and CPA would welcome constructive comments on them.

Volunteer laboratories are piloting the standards and will be assessed for compliance to the new standards at the beginning of 2002. In the second half of 2002 CPA will be retraining assessors (inspectors) to assess to the new standards. From April 2003, the revised standards will replace the current standards and assessments to the revised standards will start in October 2003.

\section{CONCLUSIONS}

The original CPA standards have now been in use for nearly 10 years and have proved to be a major influence in laboratory medicine in the UK. However, over the years anomalies have arisen in the interpretation of the standards, leading to some inconsistency at the time of inspection. The revision of the standards that has now been done aims to make compliance unequivocally verifiable at assessment, in addition to bringing them into line with the new ISO standards. In particular, the emphasis in the new standards is on the operation of a quality management system in laboratories and the authors believe that compliance with these standards will also ensure compliance with ISO standards ISO 15189, ISO 17025:1999, and ISO 


\section{Take home messages}

- New standards for accreditation of medical laboratories in the UK are compatible with the latest international standards, ISO9001:2000, ISO/IEC 17025:1999, ISO/ FDIS 15189, and the EC4 "Essential criteria for quality systems of medical laboratories"

- The needs and requirement of the users are central to the standards

- The standards provide a logical basis for managing quality and competence in the medical laboratory

- The standards will be introduced following extensive consultation with professional bodies and pilot studies

- Retraining of inspectors will aim to ensure objective assessment of medical laboratories in the UK

9001:2000. This will enable departments in the UK, and others who choose to use these standards, to have an accreditation that is recognised throughout the European Community. The detailed cross referencing of the standards to the ISO documents reinforces this point.

The standards presented here are written in a generic fashion so that that they can be applicable to all disciplines of laboratory medicine. The previous standards made reference specifically to the mortuary and blood transfusion units, but these specific areas should be capable of being assessed to the generic standards.

One of the most important aspects of these standards is the emphasis on the needs and requirements of the users of the laboratory service. This was not a feature of the first standards, and was not often a feature of the management of the medical laboratory in the past. It is hoped that this new emphasis will encourage departments to seek the opinions of those who use their services and, if appropriate, try to meet their requirements.

"Volunteer laboratories are piloting the standards and will be assessed for compliance to the new standards at the beginning of $2002^{\prime \prime}$

The aim of CPA in producing these standards, along with its scheme for externally assessing compliance with them, is to integrate the certification standards that apply to a quality management system with the accreditation standards to ensure that a medical laboratory can deliver a proper service to its users.

\section{Authors' affiliations}

D Burnett, Lindens Lodge, Bradford Place, Penarth CF64 1LA, UK C Blair, Clinical Pathology Accreditation (UK) Ltd, 45 Rutland Park, Botanical Gardens, Sheffield S10 2PB, UK
M R Haeney, Department of Immunology, Clinical Sciences Building,

Hope Hospital, Salford, Manchester M6 8HD, UK

S L Jeffcoate, 5 Elmhurst Drive, Dorking, Surrey RH4 2BA, UK

K W M Scott, Department of Pathology, New Cross Hospital,

Wolverhampton, West Midlands WV10 OQP, UK

D L Williams, Department of Clinical Biochemistry, Pathology

Laboratory, The Royal Berkshire Hospital, Reading, Berkshire RGI 5AN,

UK

\section{REFERENCES}

1 Advisory Task Force on Standards to the Audit Steering Committee of the Royal College of Pathologists. Pathology department accreditation in the United Kingdom: a synopsis. J Clin Pathol 1991;44:798-802.

2 International Organisation for Standardisation. ISO/IEC Guide 58: 1993 Calibration and testing laboratory systems-General requirements for operation and recognition. Geneva: International Organisation for Standardisation, 1993

3 International Society for Quality in Health Care. International standards for health care accreditation bodies. Victoria, Australia: International Society for Quality in Health Care, 2000.

4 CPA. Standards for the medical laboratory, Version 1.0. CPA (UK) Ltd, January 2001 (www/cpa-uk .co.uk).

5 Jansen RTP, Blaton V, Burnett D, et al. Essential criteria for quality systems in laboratory medicine. Eur J Clin Chem Clin Biochem 1997:35:121-32.

6 Jansen RTP, Blaton V, Burnett D, et al. Additional essential criteria for quality systems in laboratory medicine. Eur J Clin Chem Clin Biochem 1998;36:249-52.

7 International Organisation for Standardisation. ISO/FDIS 15189 Quality management in the medical laboratory. Geneva: International Organisation for Standardisation, August 2000

8 International Organisation for Standardisation. ISO/IEC 17025:1999 General requirements for the competence of testing and calibration laboratories. Geneva: International Organisation for Standardisation, 1999

9 International Organisation for Standardisation. ISO 9001:2000 Quality management systems-Requirements. Geneva: International Organisation for Standardisation, 2000

10 Withdrawn

11 International Organisation for Standardisation. ISO 9000:2000 Quality management systems - Fundamentals and vocabulary. Geneva: International Organisation for Standardisation, 2000.

12 International Organisation for Standardisation. ISO 9004:2000 Quality management systems-Guidelines for quality improvements. Geneva: International Organisation for Standardisation, 2000

13 International Organisation for Standardisation. ISO/IEC Guide 2 General terms and their definitions concerning standardization and related activity. Geneva: International Organisation for Standardisation, 1991.

14 International Organisation for Standardisation. ISO/IEC guide 25:1990 General requirements for the competence of calibration and testing laboratories. Geneva: International Organisation for Standardisation, 1990.

15 European Organisation for Standardisation. EN 45001:1989 General criteria for the operation of testing laboratories. Paris: European Organisation for Standardisation, 1989

16 Jansen RTP, Kenny D, Blaton V, et al. Usefulness of EC4 essential criteria for quality systems of medical laboratories as guidelines to the ISO 15189 and ISO 17025 documents. Clin Chem Lab Med 2000;38: 1057-64.

17 International Organisation for Standardisation. EN ISO/DIS 15189, Medical laboratories-Particular requirements for quality and competence. Geneva: International Organisation for Standardisation, July 2001 . 